\title{
Real time monitoring of biomolecules: dynamic response limits of affinity-based sensors
}

\author{
Rafiq M. Lubken ${ }^{1,3}$, Arthur M. de Jong ${ }^{2,3}$ \& Menno W.J. Prins ${ }^{1,2,3,4, *}$ \\ ${ }^{1}$ Department of Biomedical Engineering, Eindhoven University of Technology, Eindhoven 5612 AZ, the Netherlands. \\ ${ }^{2}$ Department of Applied Physics, Eindhoven University of Technology, Eindhoven 5612 AZ, the Netherlands. \\ ${ }^{3}$ Institute for Complex Molecular Systems (ICMS), Eindhoven University of Technology, Eindhoven 5612 AZ, the Netherlands. \\ ${ }^{4}$ Helia Biomonitoring, Eindhoven $5612 \mathrm{AZ}$, the Netherlands. \\ *email: m.w.j.prins@tue.nl.
}

SUPPLEMENTARY INFORMATION

1. Finite-element simulations

2. Concentration change rate

3. Frequency-dominated lag time

4. Expected analyte-binder complex density change

5. Precision of concentration change rate

6. Example calculations of insulin monitoring

7. References 


\section{Finite-element simulations}

The simulation study of the time-dependent behavior of the monitoring system is performed using dimensionless parameters for all mass transport processes and reaction rates. ${ }^{1,2}$ The nondimensionalized parameters for mass transport by diffusion and advection are given in Table S1.

Table S1. Dimensionless parameters used in the finite-element analysis for modeling mass transport by diffusion and advection.

\begin{tabular}{|l|l|l|}
\hline Dimensionless parameter & Symbol & Expression \\
\hline Analyte concentration in the measurement chamber & $\tilde{c}^{*}$ & $\tilde{c}^{*}=C_{a}^{*}(t) / C_{a, 0}$ \\
\hline Longitudinal distance & $\tilde{x}$ & $\tilde{x}=x / L$ \\
\hline Transversal distance & $\tilde{y}$ & $\tilde{y}=y / H$ \\
\hline Time & $\tilde{t}$ & $t=H^{2} / D \rightarrow \tilde{t}=t D / H^{2}$ \\
\hline
\end{tabular}

For all finite-element analyses, the frequency is nondimensionalized using the diffusion time $\tau_{D}$ (e.g., in Figure $3 a$ and c) and thereafter recalculated to normalize with respect to other time scales (e.g., $\tau_{R}$ in Figure $3 \mathrm{~b}$ and c). When flow is included, the used analytical expression of the flow profile is given by:

$$
\vec{v}(y)=\frac{6 Q}{W H^{3}} y(H-y) \overrightarrow{e_{x}}
$$

with $\vec{v}(y)$ being the flow speed as a function of the height $y$ inside the measurement chamber, $Q$ the flow rate, $W$ the width of the measurement chamber, and $H$ the height of the measurement chamber. The general equation used in the simulation to describe mass transport by advection and diffusion is given by:

$$
\frac{\delta C_{a}^{*}}{\delta t}=D \nabla^{2} C_{a}^{*}(t)-\vec{v}(y) \cdot \nabla C_{a}^{*}
$$

with $\frac{\delta C_{a}^{*}}{\delta t}$ being the time-derivative of the (spatial- and time-dependent) analyte concentration $C_{a}^{*}$ in the measurement chamber and $D$ the diffusion coefficient. The dimensionless form of Equation $\mathrm{S} 2$ using the defined parameters in Table S1 is derived in Box S1.

Box S1: Derivation of the dimensionless advection-diffusion equation.

$$
\begin{aligned}
& \frac{\delta\left(\tilde{c}^{*} C_{a .0}\right)}{\delta\left(\frac{\tilde{t} H^{2}}{D}\right)}=D \frac{\delta^{2}\left(\tilde{c}^{*} C_{a .0}\right)}{\delta(\tilde{x} L)^{2}}+D \frac{\delta^{2}\left(\tilde{c}^{*} C_{a .0}\right)}{\delta(\tilde{y} H)^{2}}-\frac{6 Q}{W H^{3}} \tilde{y} H(H-\tilde{y} H) \frac{\delta\left(\tilde{c}^{*} C_{a .0}\right)}{\delta(\tilde{x} L)} \\
& \frac{\delta \tilde{c}^{*}}{\delta \tilde{t}}=\frac{H^{2}}{D} \frac{1}{L^{2}} D \frac{\delta^{2} \tilde{c}^{*}}{\delta \tilde{x}^{2}}+\frac{H^{2}}{D} \frac{1}{H^{2}} D \frac{\delta^{2} \tilde{c}^{*}}{\delta \tilde{y}^{2}}-\frac{H^{2}}{D} \frac{6 Q}{W H^{3}} \frac{1}{L} \tilde{y} H^{2}(1-\tilde{y}) \frac{\delta \tilde{c}^{*}}{\delta \tilde{x}} \\
& \frac{\delta \tilde{c}^{*}}{\delta \tilde{t}}=\frac{H^{2}}{L^{2}} \frac{\delta^{2} \tilde{c}^{*}}{\delta \tilde{x}^{2}}+\frac{\delta^{2} \tilde{c}^{*}}{\delta \tilde{y}^{2}}-\frac{6 Q H}{L D W} \tilde{y}(1-\tilde{y}) \frac{\delta \tilde{c}^{*}}{\delta \tilde{x}}
\end{aligned}
$$

Using the derivation given in Box S1, the measurement chamber aspect ratio $\lambda=L / H$, and the longitudinal Péclet number $P e_{L}=\frac{Q}{\lambda D W}$ (see Table 1), the simplified dimensionless advection-diffusion equation is given by:

$$
\frac{\delta \tilde{c}^{*}}{\delta \tilde{t}}=\frac{1}{\lambda^{2}} \frac{\delta^{2} \tilde{c}^{*}}{\delta \tilde{x}^{2}}+\frac{\delta^{2} \tilde{c}^{*}}{\delta \tilde{y}^{2}}-6 P e_{L} \tilde{y}(1-\tilde{y}) \frac{\delta \tilde{c}^{*}}{\delta \tilde{x}}
$$


The nondimensionalized parameters for the reaction rate are given in Table S2.

Table S2. Dimensionless parameters used in the finite-element analysis for modeling the reaction at the sensor surface.

\begin{tabular}{|l|l|l|}
\hline Dimensionless parameter & Symbol & Expression \\
\hline Analyte concentration at the sensor surface & $\tilde{c}$ & $\tilde{c}=C_{a}(t) / C_{a, 0}$ \\
\hline Density of analyte-binder complexes & $\tilde{\gamma}$ & $\tilde{\gamma}=\gamma_{a b} /\left(C_{a, 0} H\right)$ \\
\hline Time & $\tilde{t}$ & $\tilde{t}=t D / H^{2}$ \\
\hline
\end{tabular}

The general equation used in the simulation to model the reaction at the sensor surface is given by:

$$
\frac{\delta \gamma_{a b}(t)}{\delta t}=k_{\mathrm{on}} C_{a}(t)\left[\Gamma_{b}-\gamma_{a b}(t)\right]-k_{\mathrm{off}} \gamma_{a b}(t)
$$

with $\frac{\delta \gamma_{a b}}{\delta t}$ being the time-derivative of the (spatial- and time-dependent) density $\gamma_{a b}$ of analyte-binder complexes and $C_{a}(t)$ the analyte concentration at the sensor surface, which is known by solving Equation $\mathrm{S} 3$. The dimensionless form of Equation S4 is derived in Box S2.

Box S2: Derivation of the dimensionless reactive rate equation.

$$
\begin{aligned}
& \frac{\delta\left(\widetilde{\gamma} C_{a, 0} H\right)}{\delta\left(\frac{\tilde{t} H^{2}}{D}\right)}=k_{\mathrm{on}} \tilde{C} C_{a, 0}\left(\Gamma_{b}-\tilde{\gamma} C_{a, 0} H\right)-k_{\mathrm{off}} \tilde{\gamma} C_{a, 0} H \\
& \frac{\delta \widetilde{\gamma}}{\delta \tilde{t}}=\frac{H^{2}}{D} \frac{k_{\mathrm{on}} \tilde{c}}{H}\left(\Gamma_{b}-\tilde{\gamma} C_{a, 0} H\right)-\frac{H^{2}}{D} k_{\mathrm{off}} \tilde{\gamma} \\
& \frac{\delta \widetilde{\gamma}}{\delta \tilde{t}}=\frac{k_{\mathrm{on}} \Gamma_{b} H}{D} \tilde{c}-\frac{k_{\mathrm{on}} H}{D} \tilde{c} \tilde{\gamma} C_{a, 0} H-\frac{H^{2}}{D} k_{\mathrm{off}} \tilde{\gamma} \\
& \frac{\delta \tilde{\gamma}}{\delta \tilde{t}}=\frac{C_{a, 0} H}{\Gamma_{b}} \frac{k_{\mathrm{on}} \Gamma_{b} H}{D}\left[\tilde{C}\left(\frac{\Gamma_{b}}{C_{a, 0} H}-\tilde{\gamma}\right)-\frac{K_{d}}{C_{a, 0}} \tilde{\gamma}\right]
\end{aligned}
$$

Using the derivation given in Box S2 and Damköhler number $D a=\frac{k_{\mathrm{on}} \Gamma_{b} H}{D}$ (see Table 1), the simplified dimensionless reaction rate equation is given by:

$$
\frac{\delta \widetilde{\gamma}}{\delta \tilde{t}}=\frac{C_{a, 0} H}{\Gamma_{b}} D a\left[\tilde{c}\left(\frac{\Gamma_{b}}{C_{a, 0} H}-\tilde{\gamma}\right)-\frac{K_{\mathrm{d}}}{C_{a, 0}} \tilde{\gamma}\right]
$$

The nondimensionalized parameters for the oscillating concentration are given in Table S3. 
Table S3. Dimensionless parameters used in the finite-element analysis for modeling the oscillating concentration input.

\begin{tabular}{|l|l|l|}
\hline Dimensionless parameter & Symbol & Expression \\
\hline Analyte concentration in the system of interest & $\tilde{c}_{a, 0}$ & $\tilde{c}_{a, 0}=C_{a, 0}(t) / C_{a, 0}$ \\
\hline $\begin{array}{l}\text { Mean analyte concentration in the system of } \\
\text { interest }\end{array}$ & - & $1=C_{a, 0} / C_{a, 0}$ \\
\hline Concentration change & $\Delta \tilde{C}_{a, 0}$ & $\Delta \tilde{C}_{a, 0}=\Delta C_{a, 0} / C_{a, 0}$ \\
\hline Time & $\tilde{t}$ & $\tilde{t}=t D / H^{2}$ \\
\hline Frequency & $\tilde{f}$ & $\tilde{f}=f H^{2} / D$ \\
\hline
\end{tabular}

The general equation used in the simulation to describe the oscillating concentration in the system of interest $C_{a, 0}$ is given by:

$$
C_{a, 0}(t)=C_{a, 0}+\frac{\Delta C_{a, 0}}{2} \sin (2 \pi f t+\phi)
$$

with $C_{a, 0}$ being the mean concentration in the system of interest, $\Delta C_{a, 0}$ the concentration change, $f$ the frequency, and $\phi$ the phase. The outcome of Equation S6 forms the boundary condition at the inlet of the measurement chamber when solving Equations S3 and S4 simultaneously. The dimensionless form of Equation S6 is derived in Box S3.

Box S3: Derivation of the dimensionless oscillating concentration input.

$$
\begin{aligned}
& \tilde{c}_{a, 0} C_{a, 0}=C_{a, 0}+\frac{\Delta \tilde{C}_{a, 0} C_{a, 0}}{2} \sin \left(2 \pi \tilde{f} \frac{D}{H^{2}} \tilde{t} \frac{H^{2}}{D}+\phi\right) \\
& \tilde{c}_{a, 0}=1+\frac{\Delta \tilde{C}_{a, 0}}{2} \sin (2 \pi \tilde{f} \tilde{t}+\phi) \\
& \tilde{c}_{a, 0}=1+\frac{\Delta \tilde{C}_{a, 0}}{2} \sin (2 \pi \tilde{f} \tilde{t}+\phi)
\end{aligned}
$$




\section{Concentration change rate}

The concentration change rate (CCR) is the change (increase or decrease) in concentration per unit time. From an concentration profile, the CCR can be estimated using a characteristic concentration change $\Delta C_{a, 0}$, i.e., the difference between a minimum and a maximum concentration value, and a characteristic fluctuation time $t_{\text {fluc }}$, i.e., the time required to increase from the minimum and maximum concentration value or vice versa (see Figure S1a and Figure 2):

$$
C C R=\Delta C_{a, 0} / t_{\text {fluc }}
$$

From a sinusoidal concentration profile with amplitude $\Delta C_{a, 0} / 2$ and frequency $f$, the CCR can be calculated using the concentration change $\Delta C_{a, 0}$ and the frequency $f$, where $f=1 /\left(2 \cdot t_{\text {fluc }}\right)$ (see Figure $\mathrm{S} 1 \mathrm{~b}$ ):

$$
C C R=4 \frac{\Delta C_{a, 0}}{2} f=2 \Delta C_{a, 0} f
$$
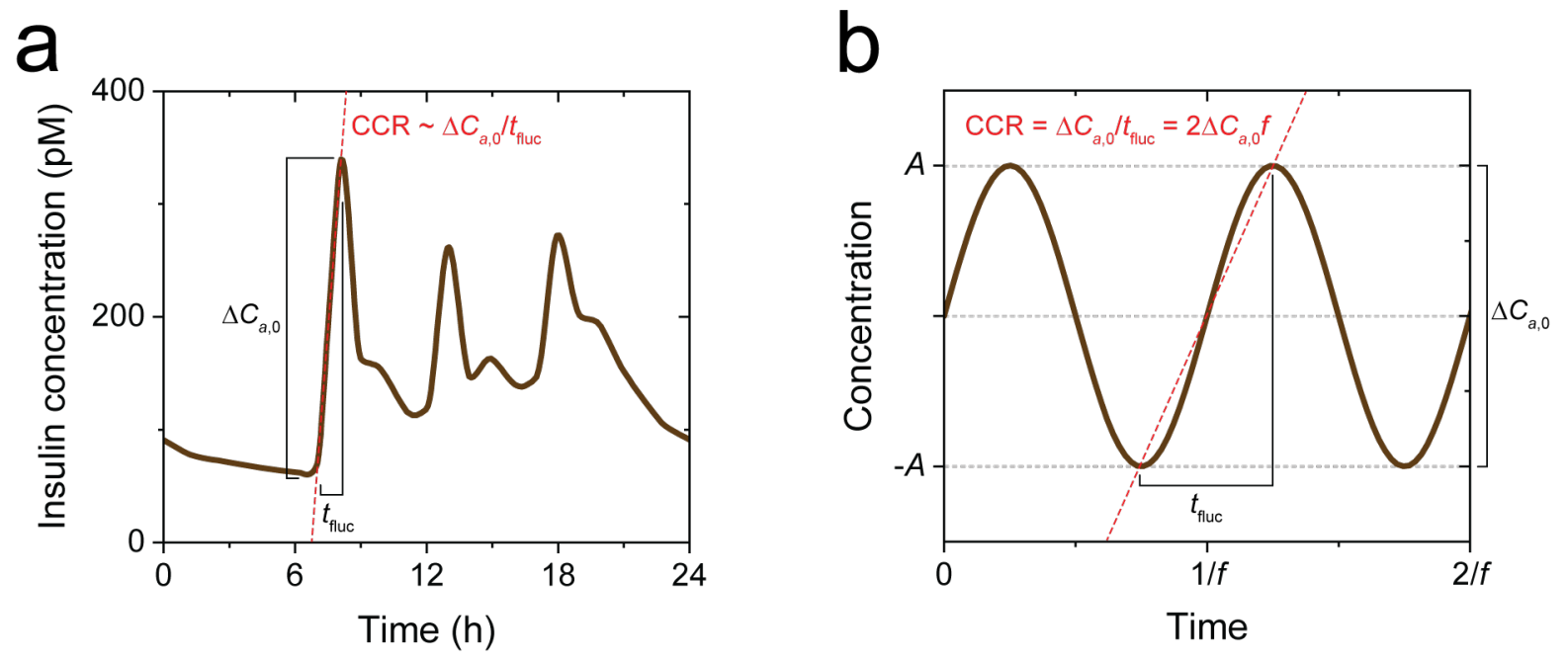

Figure S1. Insulin concentration profile and a sinusoidal function with a typical concentration change and a typical fluctuation time. (a) Insulin concentration profile (brown) with a typical concentration change $\Delta C_{a, 0}$ and a typical fluctuation time $t_{\text {fluc }}$. The concentration change rate (CCR) can be estimated by CCR $\sim \Delta C_{a, 0} / t_{\text {fluc }}$ (red dashed line). (b) Sinusoidal concentration profile with amplitude $\Delta C_{a, 0} / 2$ and frequency $f$. The CCR can be calculated by CCR $=\Delta C_{a, 0} / t_{\text {fluc }}=2 \Delta C_{a, 0} f$ (red dashed line).

The typical CCR for insulin as visualized in Figure 2 is larger than can be found in the Fourier spectra in Figure $5 \mathrm{~b}$ and $c$. The difference can be explained by the fact that in Figure $5 b$ and $c$, the observed CCR comprises multiple sinusoidal components, which results in a summed observed CCR, and thus a smaller CCR per sinusoidal concentration profile. 


\section{Frequency-dominated lag time}

In Box S4 a mathematical derivation is given for the observed lag time $\Delta t$ of the concentration $C_{a}(t)$ at the sensor surface using diffusion-based sampling, when $\Delta t$ is frequency-dominated, for a given oscillating concentration $C_{a, 0}(t)$ in the system of interest. For $f>f_{c}^{D}$, a concentration gradient in the lateral direction develops with a concentration wavelength $\lambda_{C}^{D}$.

Box S4: Mathematical derivation of the frequency-dominated lag time $\Delta t$ for the concentration $C_{a}(t)$ at the sensor surface using diffusion-based sampling.

When the observed lag time $\Delta t$ is frequency-dominated, a concentration gradient in the lateral direction exists with a characteristic length scale equal to the concentration wavelength $\lambda_{C}^{D}$ due to diffusion: $\lambda_{C}^{D}=\sqrt{D / f}$, where $D$ is the diffusion coefficient and $f$ the frequency. The phase lag $\Delta \phi^{D}$ of the observed concentration at the sensor surface compared to the concentration at the inlet of the measurement chamber, can be calculated by $\Delta \phi^{D}=$ $2 \pi H / \lambda_{c}^{D}$, while the corresponding lag time $\Delta \tau$ can be calculated by $\Delta t_{D}=\frac{\Delta \phi^{D}}{2 \pi f}=\frac{H}{f \lambda_{C}^{D}}$. This yields $\Delta t_{D}=H / \sqrt{D f}$. By normalizing $\Delta t$ and $f$ using the diffusion time scale $\tau_{D}=H^{2} / D$, we obtain $\Delta t_{D} / \tau_{D}=1 / \sqrt{f \tau_{D}}$, which results in $\Delta t_{D} \propto 1 / \sqrt{f}$ (see Figure 3a).

In Box S5 a mathematical derivation is given for the observed lag time $\Delta t$ of the density $\gamma_{a b}$ of analyte-binder complexes, when $\Delta t$ is frequency-dominated, for a given concentration $C_{a}(t)$ at the sensor surface. For $f>f_{c}^{R}$, no equilibrium can be reached resulting in a quasi-equilibrium that is determined by $f$. When a concentration $C_{a}(t)$ at the sensor surface is supplied with a high frequency, the observed phase lag $\Delta \phi$ equals $\pi / 2$ (see Figure $3 b$ ). The density $\gamma_{a b}$ of analyte-binder complexes cannot lag more than this with respect to the expected density $\gamma_{a b}^{\exp }$ since the association and dissociation rates directly depend on the concentration $C_{a}(t)$ at the sensor surface and $\gamma_{a b}$ :

$$
r_{a}=k_{\mathrm{on}} C_{a}(t)\left(\Gamma_{b}-\gamma_{a b}\right) \text { and } \quad r_{d}=k_{\mathrm{off}} \gamma_{a b}
$$

with $r_{a}$ being the association rate, $k_{\mathrm{on}}$ the association rate constant, $\Gamma_{b}$ the binder density, $r_{d}$ the dissociation rate, and $k_{\text {off }}$ the dissociation rate constant. From Equation S9 can be concluded that $r_{a}$ directly responds to a change in $C_{a}(t)$, while $r_{d}$ only responds after analyte-binder complexes are formed in the association reaction.

Box S5: Mathematical derivation of the frequency-dominated lag time $\Delta t$ for the density $\gamma_{a b}$ of analyte-binder complexes.

In contrast to diffusion, the phase lag $\Delta \phi^{R}$ of the observed density $\gamma_{a b}$ of analyte-binder complexes with respect to the expected density $\gamma_{a b}^{\text {exp }}$ of analyte-binder complexes, is independent of frequency for $f>f_{C}^{R}$ since $\Delta \phi^{R}=$ $\pi / 2$ since the association and the dissociation rates depend on the concentration $C_{a}(t)$ at the sensor surface. The lag time can be calculated by $\Delta t_{R}=\frac{\Delta \phi^{R}}{2 \pi f}$. This yields $\Delta t_{R}=\frac{1}{4 f}$. By normalizing $\Delta t$ and $f$ using the reaction time $\tau_{R}=\frac{1}{k_{\mathrm{on}} C_{a}+k_{\mathrm{off}}}$, we obtain $\Delta t_{R} / \tau_{R}=\frac{1}{4 f \tau_{R}}$, which results in $\Delta t_{R} \propto 1 / f$ (see Figure $3 \mathrm{~b}$ ).

In Box S6 a mathematical derivation is given for the observed lag time $\Delta t$ of the concentration $C_{a}(t)$ at the sensor surface using advection-based sampling, when $\Delta t$ is frequency-dominated, for a given concentration $C_{a, 0}(t)$ in the 
system of interest. For $f>f_{c}^{A}$, there exists a concentration gradient perpendicular to the velocity profile with a concentration wavelength $\lambda_{C}^{A}$.

Box S6: Mathematical derivation of the frequency-dominated lag time $\Delta t$ for the concentration $C_{a}(t)$ at the sensor surface using advection-base sampling.

When the observed lag time $\Delta t$ is frequency-dominated, an oscillating concentration gradient exists with a characteristic length scale equal to the concentration wavelength $\lambda_{C}^{A}$ due to advection: $\lambda_{C}^{A}=\frac{Q}{H W f}$, where $D$ is the diffusion coefficient and $f$ the frequency.

The phase lag $\Delta \phi^{A}$ of the observed concentration at the sensor surface compared to the concentration at the inlet of the measurement chamber, can be calculated by $\Delta \phi^{A}=2 \pi L / \lambda_{c}^{A}$, while the corresponding lag time $\Delta \tau$ can be calculated by $\Delta t_{A}=\frac{\Delta \phi^{A}}{2 \pi f}=\frac{H L W}{f \lambda_{C}^{D}}$. This yields $\Delta t_{A}=\frac{H L W}{Q}$ which equals the characteristic advection time. The frequency-dependency is caused by diffusion to the sensor surface, where the characteristic diffusion length is much smaller than measurement chamber height $H$ which results in a less strong dependency of the lag time $\Delta t$ on the frequency $f$ (compare Figures 3a and $4 a$ ). 


\section{Expected analyte-binder complex density change}

The expected analyte-binder complex density $\gamma_{a b}^{\exp }$ can be calculated for a given analyte concentration $C_{a}(t)$ at the sensor surface. For this we use the following equation:

$$
\gamma_{a b}^{\exp }=\Gamma_{b} \frac{C_{a}(t)}{C_{a}(t)+K_{d}}
$$

with $\Gamma_{b}$ being the total binder density (assumed to be $10^{-9} \mathrm{~mol} \mathrm{~m}^{-2}$, i.e., $600 \mu \mathrm{m}^{-2}$ ), $C_{a}(t)$ the analyte concentration at the sensor surface, and $K_{d}$ the dissociation constant. When $C_{a}(t)$ oscillates with a concentration change $\Delta C_{a}$ around a mean concentration $C_{a}$, this will result in an oscillating $\gamma_{a b}^{\exp }$ with a density change $\Delta \gamma_{a b}^{\exp }$. For this we use the following equation:

$$
\Delta \gamma_{a b}^{\exp }=\Gamma_{b} \frac{C_{a}+\Delta C_{a} / 2}{C_{a}+\Delta C_{a} / 2+K_{d}}-\Gamma_{b} \frac{C_{a}-\Delta C_{a} / 2}{C_{a}-\Delta C_{a} / 2+K_{d}}
$$

Under the assumption that $C_{a} \ll K_{d}, \Delta \gamma_{a b}^{\exp }$ can be calculated by:

$$
\Delta \gamma_{a b}^{\exp } \cong \Gamma_{b} \frac{\Delta C_{a}}{K_{d}}
$$




\section{Precision of concentration change rate}

To calculate the precision of the monitoring system we define a rate sensitivity which is the smallest concentration change rate (CCR) that can be quantified with an error of $10 \%$. We refer to this minimum CCR as the limit of quantification of CCR (LoCCR). In this paper, we assume that two concentrations need to be distinguished from each other within a given time span based on a sinusoidal oscillating concentration $C_{a, 0}(t)$ with mean concentration $C_{a, 0}$, concentration change $\Delta C_{a, 0}$, and frequency $f$. A mathematical derivation of the LoCCR is given in Box S7.

Box S7: Mathematical derivation of the sensitivity of the concentration change rate in a biomolecular monitoring system.

The CCR can be calculated by $\mathrm{CCR}=2 \Delta C_{a, 0} f$ assuming an oscillating concentration with concentration change $\Delta C_{a, 0}$ and frequency $f$. For a given frequency $f$, the minimum CCR that can be determined precisely is when the coefficient of variation $C V_{\mathrm{CCR}}$ of the concentration change rate is smaller than or equal to $10 \%$, i.e., $C V_{\mathrm{CCR}}=$ $C V_{\Delta C_{a, 0}}=\frac{\sigma_{\Delta C_{a, 0}}}{\Delta c_{a, 0}} \leq 10 \%$. The error of the concentration change $\sigma_{\Delta c_{a, 0}}$ can be calculated by:

$$
\sigma_{\Delta C_{a, 0}}^{2}=\left(\sigma_{C}^{\max }\right)^{2}+\left(\sigma_{C}^{\min }\right)^{2} \rightarrow \sigma_{\Delta C_{a, 0}} \cong \sqrt{2} \sigma_{C}
$$

where $\sigma_{C}^{\max }$ and $\sigma_{C}^{\min }$ are the errors of the maximum and minimum of the oscillating concentration profile respectively. For simplicity, we assume that $\sigma_{C}^{\max } \cong \sigma_{C}^{\min }=\sigma_{C}$ which is true if the precision of the sensor is Poisson-limited and $\Delta C_{a, 0}$ is small. The error of the concentration $\sigma_{C}$ can be determined using the error of the signal $\sigma_{S}$ and the slope of the dose-response curve: $\sigma_{C}=\frac{\delta c_{a, 0}}{\delta s} \sigma_{S}$ with $\frac{\delta S}{\delta c_{a, 0}}$ the concentration-derivative of the signal. The error of the signal $\sigma_{S}$ can be determined using the statistical variation $\sigma_{N_{a b}}$ in the number of analytebinder complexes:

$$
\sigma_{N_{a b}}=\sqrt{N_{a b}} \rightarrow \sigma_{S}=\frac{S}{\sqrt{N_{a b}}}
$$

where $N_{a b}$ is the total number of analyte molecules bound to binder molecules that contribute to the signal of the sensor and $S$ is the measured signal. Combining all derivations given above, gives the following expression for the minimum concentration change that can be determined precisely:

$$
\Delta C_{a, 0} \geq 10 \cdot \sigma_{\Delta C_{a, 0}} \rightarrow \Delta C_{a, 0} \geq 10 \sqrt{2} \frac{\delta C_{a, 0}}{\delta S} \frac{S}{\sqrt{N_{a b}}}
$$

and the LoCCR for a given frequency $f$ :

$$
\text { LoCCR }=20 f \sqrt{2} \frac{\delta C_{a, 0}}{\delta s} \frac{S}{\sqrt{N_{a b}}}
$$

The results of the mathematical derivation of the LoCCR given in Box S7, are visualized in Figure S2, where the monitoring system was assumed to be in equilibrium $(f \rightarrow 0)$, and limited by Poisson noise only. ${ }^{3}$ Figure S2a shows the normalized signal $S$ as a function of concentration $C_{a, 0}$, which directly scales with the fractional occupancy $f$ of binder molecules by analyte molecules. The normalized signal can be calculated using the following equation, assuming first-order Langmuir kinetics:

$$
S=A_{B}+\left(1-A_{B}\right) \frac{C_{a, 0}}{C_{a, 0}+K_{d}}
$$


with $S$ being the measured signal, $A_{B}$ the background signal, $C_{a, 0}$ the concentration in the system of interest, and $K_{d}$ the dissociation constant. Here, it was assumed that there is no background signal. The error of the signal is determined by the number of observed analyte-binder complexes on the sensor surface within a signal collection area of $A_{s}=1 \mathrm{~mm}^{2}$ with a binder molecule density $\Gamma_{b}=10^{-9} \mathrm{~mol} \mathrm{~m}^{-2}\left(600 \mu \mathrm{m}^{-2}\right)$. This error of the signal $\sigma_{S}$ is determined by the number of observed analyte-binder complexes (see Box S7) and therefore increases with an increasing $C_{a, 0}$ until saturation is reached where no more surface binders are available for binding analyte molecules (see inset, top). However, the relative error, indicated by the coefficient of variation of the measured signal $C V_{S}=$ $\sigma_{S} / S$ (see inset, bottom), decreases for increasing $C_{a, 0}$ which results in a more precise signal measurement when high concentrations are supplied to the sensor.

Figure S2b shows the coefficient of variation of the measured concentration $C V_{C}=\sigma_{C} / C_{a, 0}$ as a function of $C_{a, 0}$. The inset shows the precision of the concentration $\sigma_{C}$ that can be calculated from $\sigma_{S}$ (see Box S7). For low concentrations, the precision is Poisson-limited (dashed black line). However, for increasing concentrations, the precision starts to deviate with an increasing $C V_{C}$. This effect is caused by saturation of the binder molecules on the sensor surface decreasing the precision (i.e., increasing $C V_{C}$ ) of the concentration determination.

Figure S2c shows the minimum concentration change $\Delta C_{a, 0}$ that can be determined as a function of concentration $C_{a, 0}$. The inset shows the corresponding LoCCR (using Equation 8) as a function of frequency $f$, for a given concentration $C_{a, 0}=K_{d}$ (see Table S1). The dashed black arrow indicates the concentration as listed in Table S1. Here, it can be seen that a small $\Delta C_{a, 0}$ can be measured at low concentrations since here the absolute $\sigma_{C}$ is small. At high concentrations, $\Delta C_{a, 0}$ starts to increase steeply due to saturation effects (see panel b). A small CCR can be measured at low frequencies since CCR scales according to CCR $=2 f \Delta C_{a, 0}$.
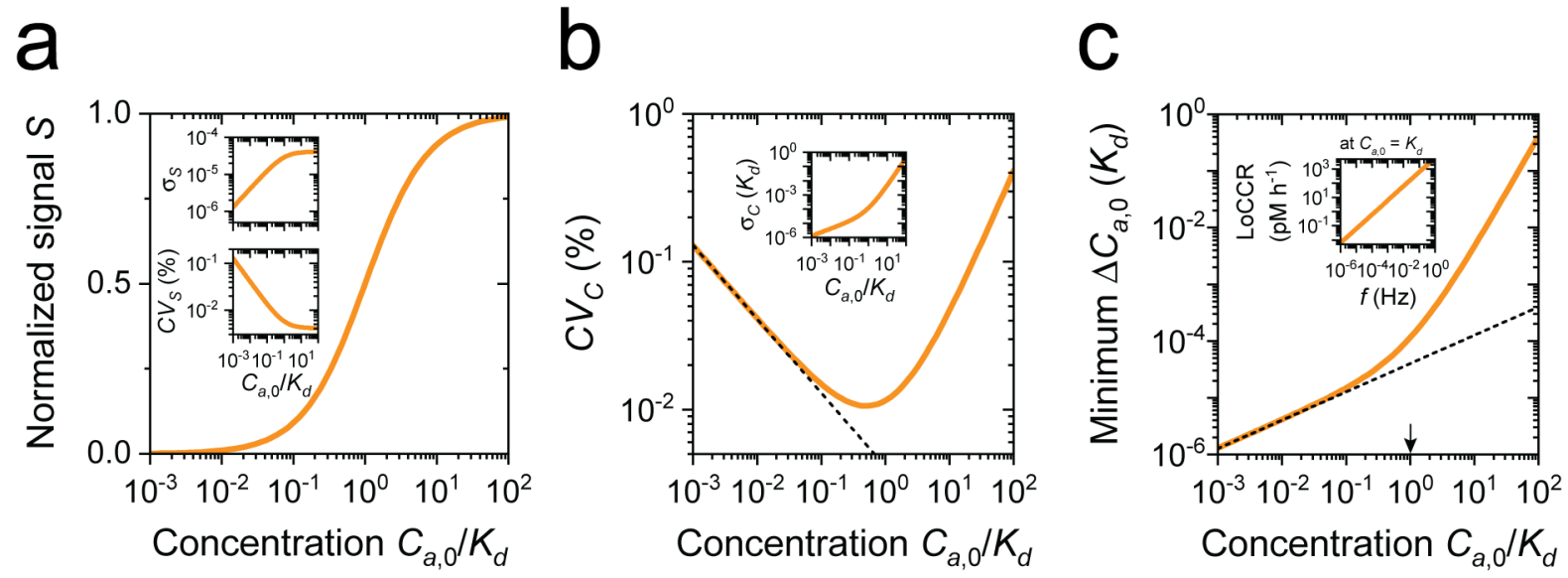

Figure S2. Precision of the signal and concentration in a monitoring system. (a) Normalized signal $S$ as function of concentration $C_{a, 0}$. The insets show the signal error $\sigma_{S}$ (top) and the coefficient of variation of the signal $C V_{S}$ (bottom) as a function of concentration $C_{a, 0}$. For low $C_{a, 0}$, the signal error is limited by the number of analyte-binder complexes. For high $C_{a, 0}$ the signal error is limited by the number of binder molecules. (b) The coefficient of variation of the concentration $C V_{C}$ as a function of mean concentration $C_{a, 0}$. The inset shows the concentration error $\sigma_{C}$ as a function of concentration. For low $C_{a}, C V_{C}$ is determined Poisson noise (black dashed line). For high $C_{a, 0}$, the precision of the concentration is decreasing by saturation of binder molecules by analyte molecules. (c) The minimum concentration change $\Delta C_{a, 0}$ that can be determined as a function of mean concentration $C_{a, 0}$. For low $C_{a, 0}$, the minimum $\Delta C_{a, 0}$ is determined by Poisson noise (black dashed line). At high $C_{a, 0}$, the minimum $\Delta C_{a, 0}$ is increasing by the saturation of binder molecules by analyte molecules. The inset shows the LoCCR as a function of frequency $f$ at a given concentration $C_{a, 0}=K_{d}$. 
From simulated data we obtain surface densities, which directly scale with signal, instead of measured concentrations. Therefore, in Figure $S 3$ the minimum observed signal change $\Delta S$ is visualized as a function of the mean concentration $C_{a, 0}$ with three signal changes $\Delta C_{a, 0} / K_{d}=50 \%, 5 \%$, and $0.5 \%$. The minimum $\Delta S$ that can be measured assuming a Poisson-limited sensor, can be estimated by the following equation (cf. Box S7):

$$
\Delta S \geq 10 \sqrt{2} \frac{S}{\sqrt{N_{a b}}}
$$

By determining the intersection (black arrows) of the dashed black line (Poisson-limit) and the orange curves that show the observed signal change $\Delta S$ as a function of the mean concentration $C_{a, 0}$ of the oscillating concentration $C_{a, 0}(t)$ for a given concentration change $\Delta C_{a, 0}$, we can estimate the minimum concentration change that can be determined at a given $C_{a, 0}$. When the frequencies are low ( $f \sim$ hours, left) the minimum concentration change $\Delta C_{a, 0}$ is small at low concentration, but for high frequencies ( $f \sim$ minutes, right), only a higher $\Delta C_{a, 0}$ can be determined precisely at that same concentration. This results in the LoCCR as visualized in Figures $5 \mathrm{a}, \mathrm{d}$ and e using Equation 8.
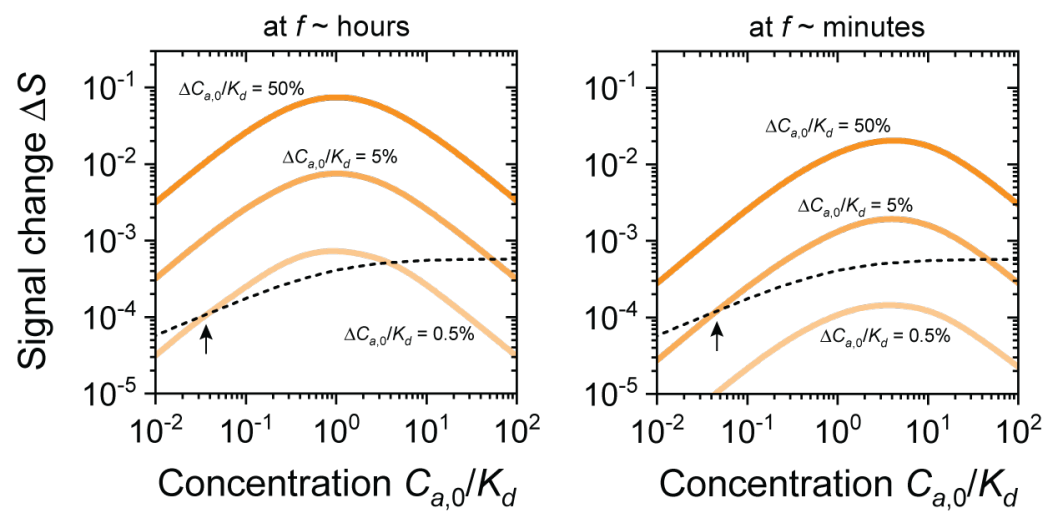

Figure S3. Minimum quantifiable concentration change at a given frequency and mean concentration of the oscillating input concentration. The signal change $\Delta S$ as a function the mean concentration $C_{a, 0}$ of an concentration profile $C_{a, 0}(t)$ at a low frequency $f(f \sim$ hours, left) and at a high $f\left(f \sim\right.$ minutes, right), for an example monitoring system with three concentration changes $\Delta C_{a, 0}$ (orange). The dashed black lines shows the minimum signal change $\Delta S$ as determined by Equation $S 14$. The intersection (black arrows) gives the minimum concentration change $\Delta C_{a, 0}$ which can be determined for a given frequency $f$ and mean concentration $C_{a, 0}$. 


\section{Example calculations of insulin monitoring}

Continuous glucose monitoring devices help diabetes patients to control their blood glucose levels. ${ }^{4,5}$ Advanced closed-loop systems are available that automatically regulate insulin delivery based on glucose monitoring data. ${ }^{6}$ Even better regulations may be enabled by also including continuous monitoring data of blood insulin levels. A typical CCR for insulin is $\sim 600 \mathrm{pM} \mathrm{h}^{-1}$ with an insulin concentration ranging from 20 to $800 \mathrm{pM}$ (see Figure 2). To reconstruct insulin concentration-time profiles as closely as possible, the LoCCR should be much smaller than 600 $\mathrm{pM} \mathrm{h}^{-1}$. Ideally, a monitoring system can measure CCRs over a range of a few decades, for instance CCR $\sim 0.6-$ $600 \mathrm{pM} \mathrm{h}^{-1}$. Assuming a sinusoidal concentration-time profile, $\mathrm{CCR}=2 \Delta C_{a, 0} f$ and $f=1 /\left(2 \cdot t_{\text {fluc }}\right) \sim 2.8 \cdot 10^{-4} \mathrm{mHz}$ (see Supplementary Note 2), typical concentration changes $\Delta C_{a, 0}$ that need to be distinguished are 0.3-300 pM, which corresponds to concentration changes of a few percent of the mean insulin concentration.

We consider the standard parameter values as listed in Table 1 with a mean insulin concentration of $100 \mathrm{pM}$. Here, we estimate the fastest fluctuation of insulin concentration profile for which the measured concentration profile still closely resembles the true concentration profile.

Diffusion-based sampling (see Figure 3): We consider a small measurement chamber height $H=200 \mu \mathrm{m}$ and a large measurement chamber height $H=800 \mu \mathrm{m}$. The diffusion coefficient of insulin is approximately $D=10^{-10} \mathrm{~m}^{2} \mathrm{~s}^{-}$ 1. For small $H(200 \mu \mathrm{m}), \tau_{D}=100 \mathrm{~s}$ and $D a \sim 1$ (neither reaction-limited nor diffusion-limited). From Figure $3 \mathrm{c}$ we obtain a cutoff frequency of $f_{c}=2 \cdot 10^{-3} \mathrm{~Hz}$. Therefore for $H=200 \mu \mathrm{m}$, insulin monitoring is reliable for a frequency $f<f_{c}$, resulting in $t_{\text {fluc }}>5 \mathrm{~min}$. For large $H(800 \mu \mathrm{m}), \tau_{D}=6400 \mathrm{~s}$ and $D a \sim 64$ (diffusion-limited). From Figure 3c we obtain a cutoff frequency of $f_{c}=1 \cdot 10^{-4} \mathrm{~Hz}$. In this case, insulin monitoring is reliable if $t_{\text {fluc }}>80 \mathrm{~min}$.

Advection-based sampling (see Figure 4): Here, we consider a high flow rate $Q=120 \mu \mathrm{L} \mathrm{min} \mathrm{m}^{-1}$ and a low flow rate $Q=0.1 \mu \mathrm{L} \mathrm{min}{ }^{-1}$. For high $Q\left(120 \mu \mathrm{L} \mathrm{min}{ }^{-1}\right), \tau_{A}=1 \mathrm{~s}$ and $P e_{L}=100$ (mass transport process is diffusion-limited). From Figure $4 \mathrm{c}$ we obtain a cutoff frequency $f_{c}=1 \cdot 10^{-2} \mathrm{~Hz}$. Accurate insulin monitoring is reliable for $f<f_{c}$, which

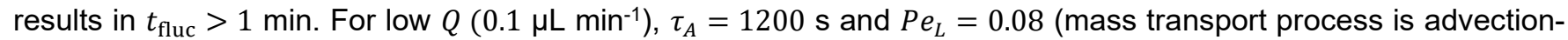
limited). From Figure $4 \mathrm{c}$ we obtain a cutoff frequency $f_{c}=8 \cdot 10^{-4} \mathrm{~Hz}$. Now accurate insulin monitoring is reliable for $f<f_{c}$, which corresponds to $t_{\text {fluc }}>10 \mathrm{~min}$. 


\section{References}

(1) Squires, T.M., Messinger, R.J. \& Manalis, S.R. Making It Stick: Convection, Reaction and Diffusion in Surface-Based Biosensors. Nature Biotechnology 26, 417-426 (2008).

(2) Lubken, R.M., Bergkamp, M.H., de Jong, A.M. \& Prins, M.W.J. Sensor for the Rapid Monitoring of Biomolecules at Low Concentrations Over Long Time Spans. ACS Sensors (2021), doi/10.1021/acssensors.1c01991.

(3) Lubken, R.M., de Jong, A.M. \& Prins, M.W.J. How Reactivity Variability of Biofunctionalized Particles Is Determined by Superpositional Heterogeneities. ACS Nano 15, 1331-1341 (2021).

(4) Rodbard, D. Continuous Glucose Monitoring: A Review of Successes, Challenges, and Opportunities. Diabetes Technology \& Therapeutics 18, 3-13 (2016).

(5) Klonoff, D.C., Ahn, D. \& Drincic, A. Continuous Glucose Monitoring: A Review of the Technology and Clinical Use. Diabetes Research and Clinical Practice 133, 178-192 (2017).

(6) Boughton, C.K. \& Hovorka, R. New Closed-Loop Insulin Systems. Diabetologia 64, 1007-1015 (2021). 